'Departamento de Nutrición, Diabetes y Metabolismo. Facultad de Medicina. Pontificia Universidad Católica de Chile. Santiago. Chile.

2UDA-Ciencias de la Salud Carrera de Nutrición y Dietética. Facultad de Medicina. Pontificia Universidad Católica de Chile. Santiago. Chile.

aNutricionista. PhD Nutrición. bBioquímico.

CPhD Biología. MSc Bioestadística.

MSc Epidemiología Genética.

Recibido el 2 de abril de 2014 aceptado el 6 de agosto de 2014

Correspondencia a: Constanza Arancibia Verdugo Teléfono: 22111664 ciaranci@uc.c coni77777@gmail.com

\section{Evaluación de la insulinemia post carga oral de glucosa como método diagnóstico de resistencia a la insulina}

\author{
CONSTANZA ARANCIBIA ${ }^{1}$, JOSÉ GALGANI ${ }^{1,2, a}$, JUAN P. VALDERAS ${ }^{1}$, \\ MAURICIO MORALES ${ }^{1, b}$, JOSÉ LUIS SANTOS ${ }^{1, \mathrm{c}}$, FELIPE POLLAK ${ }^{1}$
}

\section{Evaluation of serum insulin levels after an oral glucose load for the diagnosis of insulin resistance}

\begin{abstract}
Background: In our country, the assessment of insulin resistance (IR) measuring serum insulin levels at 60 and 120 minutes after a $75 \mathrm{~g}$ oral glucose tolerance test (OGTT), is usual. However, there is no information about the distribution of serum insulin levels in the Chilean population. Aim: To assess the distribution of serum insulin levels at 60 and 120 minutes during OGTTs and suggest a statistical cut-off point to estimate the degree of IR. Material and Methods: Retrospective analysis of 1815 OGTTs performed in non-diabetic subjects aged between 18 and 75 years, at a university medical center. HOMA-IR (Homeostasis Model Assessment), insulin sensitivity index of Matsuda (ISI-Composite), and their correlation with serum insulin levels at 60 and 120 minutes were calculated. Results: The 75th percentiles for serum insulin levels at 60 and 120 minutes were 127 and $81 \mu \mathrm{U} / \mathrm{mL}$, respectively. There was a high correlation between HOMA-IR and ISI-Composite $(r=-089, p<0.001)$. There was a weaker although significant correlation between HOMA-IR and ISI-Composite and insulin levels at $60(r=0.56$ and -0.79 respectively, $p<0,001)$ and 120 minutes $(r=0.54$ and -0.75 respectively, $p<0,001)$. Conclusions: We propose 60 and 120 min serum insulin levels of 130 and $80 \mu \mathrm{U} / \mathrm{mL}$ respectively, as cut-off values for normality during OGTT in Chilean normoglycemic individuals.
\end{abstract}

(Rev Med Chile 2014; 142: 1106-1112)

Key words: Glucose tolerance test; Hyperinsulinism; Insulin resistance.
S e define como resistencia a la insulina (RI) a la disminución de la acción biológica de esta hormona en el organismo. Este defecto produce alteraciones en sus acciones metabólicas (homeostasis de la glucosa, metabolismo lipídico y proteico) y no metabólicas (exacerbación de efectos mitogénicos). En la ausencia de una falla de la célula beta, el páncreas compensa esta situación aumentando la secreción de insulina generando un estado de hiperinsulinemia.

La evaluación de la RI es actualmente una preocupación y necesidad de investigadores, epidemiólogos y clínicos dada su asociación con entidades clínicas tales como diabetes mellitus tipo 2 (DM 2), hipertensión arterial, dislipidemia, hiperuricemia, esteatosis hepática, síndrome de ovario poliquístico y síndrome metabólico ${ }^{1,2}$. Entre los métodos disponibles, para evaluar la RI, el clamp peuglicémico-hiperinsulinémico es método gold standard ${ }^{3}$. Sin embargo, dadas las dificultades técnicas de este método, ha sido reservado para fines de investigación. En este contexto existen otros métodos de mayor aplicabilidad clínica destacando entre ellos la prueba de tolerancia oral a la glucosa con insulinemias (PTGO). Este test consiste en la medición de insulinemias y 
glicemias en ayuno y a los 30, 60, 90 y $120 \mathrm{~min}$ posterior a la ingesta de una carga oral de $75 \mathrm{~g}$ de glucosa. Originalmente fue diseñado para clasificar a los individuos según su grado de tolerancia oral a la glucosa ${ }^{4}$, sin embargo, los resultados de las insulinemias son utilizados para estimar la RI de los individuos.

A partir de la glicemia e insulinemia de ayuno es posible calcular índices de RI, tales como HOMA-IR (Homeostatic Model Assessment) propuesto por Matthews et al. ${ }^{5} \mathrm{y}$ de los valores post carga, el índice de sensibilidad a la insulina (ISI-composite) de Matsuda y De Fronzo ${ }^{6}$. Ambos índices han mostrado una correlación significativa con el método del clamp euglicémico-hiperinsulinémico. El ISI-composite ha mostrado mayor correlación que el HOMA-IR ya que al considerar el promedio de insulinemias y glicemias obtenidos de la PTGO representa una mejor estimación de la RI corporal total, mientras que el HOMA-IR representa principalmente la RI hepática ${ }^{6,7}$.

En nuestro medio es frecuente realizar el diagnóstico de RI tomando como punto de corte insulinemias mayores a 100 y $60 \mathrm{mU} / \mathrm{mL}$ a los 60 y $120 \mathrm{~min}$, respectivamente. El primer punto de corte $(100 \mathrm{mU} / \mathrm{mL})$ se ha establecido arbitrariamente en la práctica clínica. El segundo punto de corte se propuso basado en el estudio prospectivo de Paris que asoció insulinemias mayores a $445 \mathrm{pmol} / \mathrm{l}$ (equivalente a $60 \mathrm{mU} / \mathrm{mL}$ ), con un aumento de 1,6 veces del riesgo cardiovascular independiente de las glicemias ${ }^{8}$. Esta práctica trae implicancias diagnósticas y terapéuticas que no han sido consensuadas ni tampoco validadas en nuestra población, tomando en consideración que dicho estudio fue realizado en 7.028 hombres (6.903 no diabéticos y 125 diabéticos) entre 43 y 54 años y pertenecientes a una etnia diferente.

Paralelamente, debe mencionarse la gran variabilidad en los valores de insulinemias obtenidos a partir de una misma muestra de sangre dependiendo del tipo de kit, laboratorio y muestra de sangre (sérica o plasmática) que ha sido observada en algunos estudios ${ }^{9,10,11}$, dificultando aún más la interpretación de estos valores.

El objetivo de este estudio es identificar la distribución de los valores de insulinemias plasmáticas a los 60 y 120 min en una población de 1.815 individuos que se realizaron PTGO en la Red de Salud de la Pontificia Universidad Católica de Chile (PUC), observar cómo se correlacionan estos valores con índices de RI previamente validados (HOMA-IR e ISI-composite) y de este modo establecer un punto de corte estadístico para estos valores que sirva de referencia para estimar el grado de RI en nuestra población.

\section{Material y Método}

\section{Diseño del estudio y sujetos}

Para este estudio se consideraron las PTGO con insulinemias realizadas entre octubre de 2009 y diciembre de 2011 en diferentes unidades de toma de muestra de la Red de Salud de la Pontificia Universidad Católica de Chile (PUC). Todas las muestras de sangre se procesaron en el Laboratorio Central e informados en el Departamento de Nutrición, Diabetes y Metabolismo de nuestra universidad. El estudio fue aprobado por el Comité de Ética de la Facultad de Medicina de la PUC.

En total, se tuvo acceso a 1.868 PTGO. Se incluyeron las PTGO de pacientes de edades entre 18 y 75 años. Se excluyeron mujeres embarazadas, individuos con diagnóstico previo de DM, 50 diabéticos diagnosticados por la PTGO y tres sujetos que presentaron valores de glicemia en ayuno menor a $50 \mathrm{mg} / \mathrm{dl}$. Finalmente, se dispuso de 1.815 PTGO de individuos independientes. Las características de los mismos se muestran en la Tabla 1. Los individuos fueron clasificados según su control glicémico en: normales, glicemia de ayuno alterada (IFG), intolerancia a la glucosa (IGT) e IFG + IGT, según los criterios de la ADA $2010^{12}$.

\section{Prueba de tolerancia oral a la glucosa (PTGO)}

Los pacientes acudieron a una de las unidades de toma de muestra de la PUC posterior a un ayuno nocturno de 8 a $12 \mathrm{~h}$. Antes de iniciar el examen se determinó el peso y estatura según normas de la encuesta nacional de salud 2009-2010 ${ }^{13}$. Una vez instalada la vía venosa, se extrajo una muestra de sangre para determinar la insulinemia y glicemia de ayuno. Luego, se administró por vía oral una solución de glucosa comercial $(75 \mathrm{~g}$ en $290 \mathrm{ml}$ de agua) que debió beberse en menos de 5 min. Los pacientes debían permanecer en reposo, sin fumar y sin ingerir alimentos durante el examen. Se extrajeron las muestras de sangre a los 30, 60, 90 y 120 min posterior al término de la ingesta de la solución glucosada. Las muestras de sangre para medición de glicemia e insulinemia, se recolectaron en tubos que fueron centrifugadas para la 
Insulinemia post carga de glucosa y resistencia a la insulina - C. Arancibia et al

Tabla 1. Características de los individuos según grado de tolerancia a la glucosa

\begin{tabular}{|lcccc|}
\hline & Normal & IFG & IGT & IFG + IGT \\
N (H/M) & $1.493(236 / 1.257)$ & $116(48 / 68)$ & $162(29 / 133)$ & $44(13 / 31)$ \\
\hline Edad (años) & $38 \pm 13^{\mathrm{a}}$ & $51 \pm 11^{\mathrm{b}}$ & $44 \pm 14^{\mathrm{c}}$ & $54 \pm 12^{\mathrm{b}}$ \\
$\mathrm{IMC}\left(\mathrm{kg} / \mathrm{m}^{2}\right)$ & $26 \pm 4^{\mathrm{a}}$ & $30 \pm 5^{\mathrm{b}}$ & $28 \pm 5^{\mathrm{b}}$ & $29 \pm 5^{\mathrm{b}}$ \\
Glicemia Basal (mg/dl) & $85 \pm 7^{\mathrm{a}}$ & $105 \pm 5^{\mathrm{b}}$ & $89 \pm 6^{\mathrm{c}}$ & $107 \pm 6^{\mathrm{b}}$ \\
Glicemia 120 min (mg/dl) & $93 \pm 23^{\mathrm{a}}$ & $104 \pm 22^{\mathrm{b}}$ & $156 \pm 13^{\mathrm{c}}$ & $159 \pm 14^{\mathrm{c}}$ \\
\hline
\end{tabular}

Los valores representan las medias \pm 1 DS. Valores con superíndices diferentes son significativamente diferentes $(p<0,001)$.

obtención de plasma y suero respectivamente. Las muestras obtenidas se mantuvieron entre $2-8^{\circ} \mathrm{C}$ para ser analizadas el mismo día.

\section{Análisis en sangre}

La concentración de glucosa plasmática se determinó mediante la técnica de glucosa hexoquinasa con un equipo Cobas 8000 (Roche/ Hitachi, Mannheim, Alemania). El coeficiente de variación $(\mathrm{CV})$ intra- e inter-ensayo corresponde a 0,7 y $1,2 \%$, respectivamente. La concentración de insulina sérica se determinó mediante un inmunoensayo de quimioluminiscencia con un equipo Advia Centaur XP (Siemens, Atlanta, EEUU). El CV intra- e inter-ensayo es de 4,6 y $5,9 \%$, respectivamente.

\section{Estimación del grado de resistencia a la insulina (RI)}

La RI se determinó mediante el índice HOMAIR utilizando la fórmula publicada por Matthews et al. ${ }^{14}$ : (insulinemia de ayuno, $\mathrm{mU} / \mathrm{mL} \times$ glicemia de ayuno, mg/dL/405. Alternativamente, se determinó el índice ISI-Composite mediante la fórmula propuesta por Matsuda y De Fronzo : $10.000 /$ ([glicemia de ayuno, $\mathrm{mg} / \mathrm{dL} \times$ insulinemia de ayuno, $\mathrm{mU} / \mathrm{mL}] \times$ [promedio de glicemias $(30-$ 120) de la PTGO, mg/dL $\times$ promedio de insulinemias (30-120) de la PTGO, $\mathrm{mU} / \mathrm{mL}])^{0,5}$. En este estudio se definió como resistentes a la insulina a aquellos individuos con un ISI-Composite menor al percentil 25 de todo el grupo estudiado $(n=$ 1815) tal y como se determinó en el estudio de Petersen et al. ${ }^{15}$.

\section{Análisis estadístico}

Los análisis fueron realizados mediante el programa SAS versión 9.2 (SAS Institute, Cary, $\mathrm{NC}$ ). Los datos se presentan en media \pm desviación estándar (DE) y/o percentiles según corresponda. El análisis de normalidad se realizó mediante el test de Shapiro-Wilk. Las proporciones por sexo entre grupos se evaluaron mediante análisis de $\chi^{2}$. Las diferencias entre grupos para variables continuas se determinaron mediante análisis de varianza (PROC MIXED) y las diferencias específicas mediante test de Tukey. La asociación entre variables se determinó mediante test de Spearman. El nivel de significación se fijó en 5\%.

\section{Resultados}

\section{Características de los individuos}

De la muestra estudiada $82 \%$ correspondió a mujeres y $18 \%$ a hombres. Se observaron diferencias en las edades entre los diferentes grupos según tolerancia a la glucosa, excepto entre IFG e IFG + IGT (P < 0,001; Tabla 1); 36\% de los sujetos tuvo un IMC normal, $40 \%$ sobrepeso, $23 \%$ obesidad y menos de 1\% bajo peso. El IMC resultó ser menor en el grupo normal respecto a los demás grupos $(\mathrm{P}<0,001$; Tabla 1$)$. En relación a la tolerancia oral a la glucosa observada en el grupo completo, la mayoría de los sujetos tuvieron una tolerancia normal a la glucosa (82\%), mientras que 6,9 y $2 \%$ estuvieron incluidos en el grupo IFG, IGT e IFG + IGT, respectivamente.

\section{Resistencia a la insulina}

La distribución por percentiles de las insulinemias a los 60 y 120 min y los índices HOMAIR e ISI-Composite para el total de la muestra se presenta en la Figura 1. En el total de la muestra el valor del p75 fue 137 y $95 \mathrm{mU} / \mathrm{mL}$ para las insulinemias a los 60 y $120 \mathrm{~min}$, respectivamente (Figura la y 1b). Para los índices de RI calculados, el p75 del HOMA-IR fue 3,01 y el p25 para el ISICompositefue 2,32 (Figura 1c y 1d). 


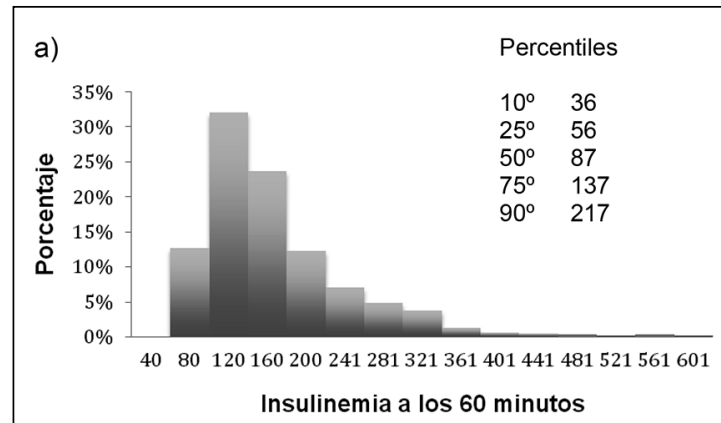

b)

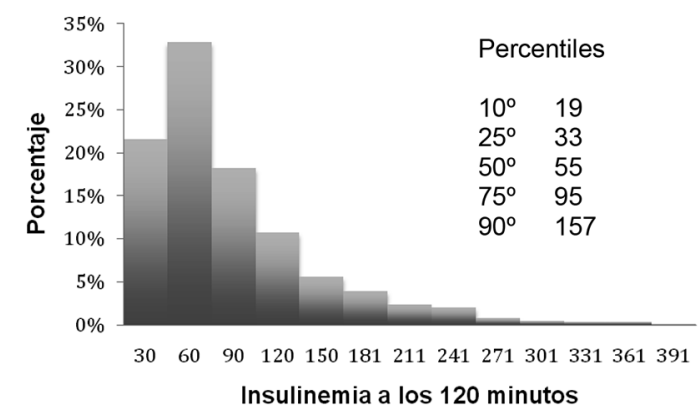

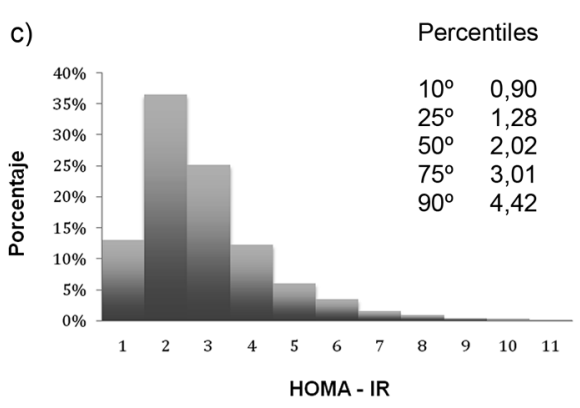

d)

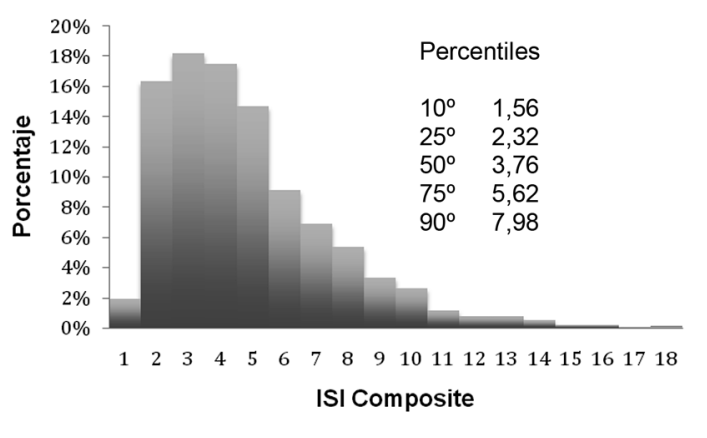

Figura 1. Distribución de insulinemias a los 60 (a), 120 min (b), HOMA-IR (c) e ISI Composite (d) en el total de los individuos.

En la Tabla 2 se puede observar la distribución por percentiles de las insulinemias a los 60 y 120 min según grado de tolerancia oral a la glucosa. Para los individuos normales, el p75 para la insulinemia a los 60 y 120 min y HOMA-IR fue 127 $\mathrm{mU} / \mathrm{mL}, 81 \mathrm{mU} / \mathrm{mL}$ y 2,76 respectivamente. El p25 para el ISI-Composite fue 2,71.

Al categorizar al total de los individuos según el ISI-Composite en resistentes $(<2,32)$ o sensibles $(>2,32)$ a la insulina, se observó que valores similares a los utilizados en la actualidad para hacer el diagnóstico de RI, esto es 101 y $67 \mathrm{mU} /$ $\mathrm{mL}$ para insulinemias a los 60 y $120 \mathrm{~min}$. Es de destacar que estos valores corresponden al p75 de los individuos sensibles a la insulina. (Tabla 3). El resto de la distribución por percentiles se puede observar en la misma Tabla.

\section{Asociación entre índices de RI e insulinemias}

En la muestra total, la insulinemia a los $60 \mathrm{~min}$

Tabla 2. Insulinemias e índices de RI para sujetos con normoglicemia y sujetos con disglicemia

\begin{tabular}{|llccccc|}
\hline & & p 10 & p 25 & p 50 & p 75 & p 90 \\
Normoglicémicos $(\mathrm{n}=1.493)$ & Ins 60 (uU/ml) & 36 & 54 & 83 & 127 & 200 \\
& Ins 120 (uU/ml) & 17 & 31 & 50 & 81 & 126 \\
& HOMA-IR & 0,88 & 1,21 & 1,89 & 2,76 & 3,85 \\
Disglicémicos ( $\mathrm{n}=322)$ & ISI (Composite) & 1,86 & 2,71 & 4,13 & 6,05 & 8,38 \\
& Ins 60 (uU/ml) & 50 & 73 & 113 & 183 & 260 \\
& Ins 120 (uU/ml) & 33 & 59 & 102 & 172 & 227 \\
& HOMA-IR & 1,23 & 1,76 & 3,14 & 4,45 & 6,13 \\
& ISI (Composite) & 1,11 & 1,49 & 2,18 & 3,29 & 4,61 \\
\hline
\end{tabular}

Los valores representan los respectivos percentiles. (Ins: insulina; 60: a los 60 min; 120: a los 120 min). 
Tabla 3. Insulinemias e índices de RI para sujetos con RI y no RI según ISI Composite

\begin{tabular}{|llccccc|}
\hline & & p 10 & p 25 & p 50 & p 75 & p 90 \\
RI según ISI composite ( $\mathrm{n}=454)$ & Ins $60(\mathrm{uU} / \mathrm{ml})$ & 94 & 132 & 183 & 242 & 300 \\
& Ins 120 (uU/ml) & 56 & 90 & 127 & 187 & 245 \\
& HOMA-IR & 2,63 & 3,22 & 4,00 & 5,13 & 6,62 \\
& ISI (Composite) & 1,07 & 1,34 & 1,68 & 2,02 & 2,20 \\
No RI según ISI composite ( $\mathrm{n}=1.361)$ & Ins $60(\mathrm{uU} / \mathrm{ml})$ & 34 & 48 & 72 & 101 & 134 \\
& Ins120 (uU/ml) & 15 & 28 & 45 & 67 & 94 \\
& HOMA-IR & 0,84 & 1,12 & 1,60 & 2,28 & 2,84 \\
& ISI (Composite) & 2,73 & 3,38 & 4,51 & 6,40 & 8,68 \\
\hline
\end{tabular}

Los valores representan los respectivos percentiles. (RI: resistencia a la insulina; Ins: insulina; 60: a los 60 min; 120: a los $120 \mathrm{~min})$.

Tabla 4. Correlación entre índices de RI derivados de la PTGO con insulinemias para el total de sujetos de la muestra

\begin{tabular}{|c|c|c|c|}
\hline & $\begin{array}{c}\text { Insulina } 60 \mathrm{~min} \\
(\mathrm{uU} / \mathrm{ml})\end{array}$ & $\begin{array}{c}\text { Insulina } 120 \mathrm{~min} \\
(\mathrm{uU} / \mathrm{ml})\end{array}$ & HOMA-IR \\
\hline Insulina 60 min (uU/ml) & 1 & - & - \\
\hline Insulina 120 min (uU/ml) & $0,609 *$ & 1 & - \\
\hline HOMA-IR & $0,558^{*}$ & $0,537^{*}$ & 1 \\
\hline ISI (composite) & $-0,790 *$ & $-0,753^{*}$ & $-0,885^{*}$ \\
\hline
\end{tabular}

Los datos corresponden a índices de correlación r. * $\mathrm{p}<0,005$ para todos los valores.

mostró una correlación positiva con la insulinemia a los $120 \min (r=0,61, P<0,005)$. Por otra parte, el HOMA-IR con la insulinemia a los $60 \mathrm{y}$ 120 min también mostraron una relación positiva ( $\mathrm{r}=0,56$ y 0,54 , respectivamente $\mathrm{P}<0,005$; Tabla 4). En cuanto al ISI-Composite este mostró una correlación negativa con la insulinemia a los $60 \mathrm{y}$ $120 \min (\mathrm{r}=-0,79$ y $-0,75$, respectivamente $\mathrm{P}<$ 0,005; Tabla 4). El ISI-Composite con el HOMAIR mostraron una fuerte correlación inversa $(\mathrm{r}=$ $-0,89 ; \mathrm{P}<0,005$; Tabla 4).

\section{Discusión}

En nuestro medio la medición de insulinemias postcarga oral de glucosa es, pese a las recomendaciones, un método frecuente para el diagnóstico de RI. A través de este examen se ha asociado a los niveles elevados de insulinemia con el riesgo de presentar DM y/o enfermedad cardiovascular en estudios prospectivos. En el estudio de Zavaroni et al. ${ }^{16}$, en forma similar al estudio prospectivo de
Paris, se observó que los individuos con insulinemias de ayuno en el cuartil superior desarrollaron hipertensión arterial, DM tipo 2 y cardiopatía coronaria. Sin embargo, en ambos estudios no se describe la distribución de las insulinemias en dichas poblaciones.

En nuestro estudio se determinó la distribución de insulinemias a los 60 y $120 \mathrm{~min}$ en una población de sujetos con diferentes grados de tolerancia a la glucosa. Se fijó el p75 para estas variables y para el HOMA-IR, así como el percentil 25 para el ISI-Composite en el total de sujetos de la muestra, y en aquellos con tolerancia a la glucosa normal, basado en estudios que han demostrado que sujetos con HOMA-IR en el cuartil superior o bien ISI-Composite en el cuartil inferior tienen un riesgo elevado de presentar comorbilidades comúnmente asociadas a la $\mathrm{RI}^{15-16}$. De este modo se determinó un valor de 137 y $95 \mathrm{mU} / \mathrm{mL}$ para insulinemias a los 60 y 120 min en el total de sujetos de la muestra y valores de 127 y $81 \mathrm{mU} / \mathrm{mL}$ para aquellos con tolerancia a la glucosa normal. Los valores entre la muestra total o normales no 
Insulinemia post carga de glucosa y resistencia a la insulina - C. Arancibia et al

difieren mayormente, dado que la mayoría de los sujetos de la muestra total tenían tolerancia a la glucosa normal $(\mathrm{n}=1493)$.

Dado que el ISI-composite ha demostrado la mejor correlación con el clamp euglicémicohiperinsulinémico para el diagnóstico de $\mathrm{RI}^{6}$ se determinó, al igual que en el estudio de Petersen et al., que sujetos con valores de este índice bajo el p25 $(2,32)$ fueran considerados como RI. En nuestra muestra se observó que, valores similares a los utilizados actualmente para hacer el diagnóstico de RI, 101 y $67 \mathrm{mU} / \mathrm{mL}$ para insulinemias a los 60 y $120 \mathrm{~min}$, corresponden al p75 de los individuos sensibles a la insulina. Esto significa que, siguiendo la práctica clínica habitual, 25\% de los sujetos de nuestra muestra y que tienen sensibilidad a la insulina normal serían diagnosticados como RI, lo que recalca la importancia de revisar el uso de estos valores.

Con respecto a cómo se correlacionan los valores de insulinemia con el HOMA-IR e ISIComposite en el total de la muestra, se observó una alta correlación de estos dos últimos índices $(r=-0,89$; Tabla 4), comparable a la observada por Matsuda et al. $(r=0,69 ; \mathrm{P}<0,001)$ en el total de una muestra de 153 sujetos con diferentes grados de tolerancia a la glucosa ${ }^{7}$. En cuanto a la correlación del ISI-Composite con insulinemias a los $60 \mathrm{y}$ 120 min esta demostró ser menor $(r=-0,79$ y $r=$ $-0,75$ respectivamente; Tabla 4 ). Más débil resultó la correlación del HOMA-IR con insulinemias a los 60 y $120 \mathrm{~min}(\mathrm{r}=0,56$ y $\mathrm{r}=0,54$, respectivamente; Tabla 4). Estos resultados son esperables considerando primero: las limitaciones técnicas con respecto a la medición de insulina en sangre, y segundo: que los valores de insulinemia elevados post carga oral de glucosa si bien representan una repuesta compensatoria a un estado de RI, no corresponden a índices de RI propiamente tal pues no consideran las variaciones que suceden concomitantemente en las glicemias, como sí lo hacen el HOMA-IR y el ISI-Composite.

Finalmente, nuestro estudio tiene algunas debilidades. En primer lugar, la muestra corresponde a sujetos que se realizaron PTGO a solicitud de su médico tratante, y no a una población de voluntarios sanos seleccionados aleatoriamente. En segundo lugar, una gran proporción de sujetos tenían sobrepeso u obesidad, eran mujeres y además se incluyeron adultos mayores, por lo que la prevalencia de hiperinsulinemia puede ser mayor. Como fortaleza está el gran tamaño de la muestra, la reproducibilidad y uniformidad del método que se utilizó para determinar la glicemia e insulinemia plasmática.

En conclusión, a partir de nuestros resultados, proponemos utilizar como punto de corte para estimar la RI en nuestra población, los valores de 130 y $80 \mathrm{mU} / \mathrm{mL}$, correspondientes a una aproximación de los 127 y $81 \mathrm{mU} / \mathrm{mL}$ para insulinemias a los 60 y 120 min de una PTGO en individuos normoglicémicos. Debe especificarse que estos valores son aplicables para el método de detección de insulina empleado en nuestro laboratorio y que sus resultados deben ser evaluados conjuntamente con el cuadro clínico.

\section{Referencias}

1. Salazar MR, Carbajal HA, Espeche WG, Dulbecco CA, Aizpurúa M, Marillet AG, et al. Relationships among insulin resistance, obesity, diagnosis of the metabolic syndrome and cardio-metabolic risk. Diab Vasc Dis Res 2011; 8 (2): 109-16.

2. López G. Consenso elaborado por la Sociedad Chilena de Endocrinología y Diabetes sobre Resistencia a la Insulina y Síndrome Metabólico. Aspectos Clínicos y terapéuticos. Rev Chil Endocrinol diabetes 2008; 1 (4): 272-81.

3. De Fronzo RA, Tobin JD, Andres R. Glucose clamp technique: a method for quantifying insulin secretion and resistance. Am J Physiol 1979; 237 (3): 214-23.

4. Hansen T, Drivsholm T, Urhammer SA, Palacios RT, Vølund A, Borch-Johnsen $\mathrm{K}$ et al. The BIGTT test: a novel test for simultaneous measurement of pancreatic beta-cell function, insulin sensitivity, and glucose tolerance. Diabetes Care 2007; 30 (2): 257-62.

5. Wallace TM, Levy JC, Matthews DR. Use and abuse of HOMA modeling. Diabetes Care 2004; 27 (6): 1487-95.

6. Matsuda M, De Fronzo RA. Insulin sensitivity indices obtained from oral glucose tolerance testing: comparison with the euglycemic insulin clamp. Diabetes Care 1999; 22 (9): 1462-70.

7. Stumvoll M, Mitrakou A, Pimenta W, Jenssen T, YkiJärvinen $\mathrm{H}$, Van Haeften $\mathrm{T}$ et al. Use of the oral glucose tolerance test to assess insulin release and insulin sensitivity. Diabetes Care 2000; 23 (3): 295-301.

8. Fontbonne AM, Eschwège EM. Insulin and cardiovascular disease. Paris Prospective Study. Diabetes Care 1991; 14 (6): 461-9.

9. Robbins DC, Andersen L, Bowsher R, Chance R, Dinesen B, Frank B, et al. Report of the American Diabetes 
Association's Task Force on standardization of the insulin assay. Diabetes 1996; 45 (2): 242-56.

10. Manley SE, Stratton IM, Clark PM, Luzio SD. Comparison of 11 human insulin assays: implications for clinical investigation and research. Clin Chem 2007; 53 (5): $922-$ 32.

11. Staten MA, Stern MP, Miller WG, Steffes MW, Campbell SE. Insulin assay standardization: leading to measures of insulin sensitivity and secretion for practical clinical care. Diabetes Care 2010; 33 (1): 205-6.

12. Diagnosis and Classification of Diabetes Mellitus. Diabetes Care 2010; 33 (Suppl 1): s62-s69.

13. Encuesta Nacional de Salud Chile. ENS 2009-2010. Tomo III: Metodología. Recuperado de http://webminsal.cl en julio de 2014 .
14. Matthews DR, Hosker JP, Rudenski AS, Naylor BA, Treacher DF, Turner RC. Homeostasis model assessment: insulin resistance and beta-cell function from fasting plasma glucose and insulin concentrations in man. Diabetologia 1985; 28 (7): 412-9.

15. Petersen KF, Dufour S, Feng J, Befroy D, Dziura J, Dalla Man C, et al. Increased prevalence of insulin resistance and nonalcoholic fatty liver disease in Asian-Indian men. Proc Natl Acad Sci USA 2006; 103 (48): 18273-7.

16. Zavaroni I, Bonini L, Gasparini P, Barilli AL, Zuccarelli A, Reaven GM. Hyperinsulinemia in a normal population as a predictor of non-insulin-dependent diabetes mellitus, hypertension, and coronary heart disease: the Barilla factory revisited. Metabolism 1999; 48 (8): 98994. 